\title{
New Cryptanalysis of Irregularly Decimated Stream Ciphers
}

\author{
Bin Zhang \\ Laboratory of Algorithmics, Cryptology and Security, \\ University of Luxembourg, \\ 6, rue Coudenhove-Kalergi, L-1359, Luxembourg \\ bin.zhang@uni.lu
}

\begin{abstract}
In this paper we investigate the security of irregularly decimated stream ciphers. We present an improved correlation analysis of various irregular decimation mechanisms, which allows us to get much larger correlation probabilities than previously known methods. Then new correlation attacks are launched against the shrinking generator with Krawczyk's parameters, LILI-U, DECIM ${ }^{v 2}$ and DECIM-128 to access the security margin of these ciphers. We show that the shrinking generator with Krawczyk's parameters is practically insecure; the initial internal state of LILI- $\amalg$ can be recovered reliably in $2^{72.5}$ operations, if $2^{24.1}$-bit keystream and $2^{74.1}$-bit memory are available. This disproves the designers' conjecture that the complexity of any divide-and-conquer attack on LILI- $\amalg$ is in excess of $2^{128}$ operations and requires a large amount of keystream. We also examine the main design idea behind DECIM, i.e., to filter and then decimate the output using the ABSG algorithm, by showing a class of correlations in the ABSG mechanism and mounting attacks faster than exhaustive search on a 160-bit (out of 192-bit) reduced version of $\mathrm{DECIM}^{v 2}$ and on a 256-bit (out of 288-bit) reduced version of DECIM-128. Our result on DECIM is the first nontrivial cryptanalytic result besides the time/memory/data tradeoffs. While our result confirms the underlying design idea, it shows an interesting fact that the security of DECIM rely more on the length of the involved LFSR than on the ABSG algorithm.
\end{abstract}

\section{Introduction}

Irregular decimation (or irregular clocking) is a well-known strategy in hardwareoriented stream cipher design. It is commonly believed that such a mechanism can strengthen the security of the underlying pseudo-random bit generators with respect to correlation [4, 5, 20, 23, 27] and algebraic attacks [9, 10].

In this paper we consider four well-known stream ciphers using irregular decimation as the main protective mechanism, namely, the shrinking generator (SG) with Krawczyk's parameters [8, 19], LILI-Ш [7], DECIM ${ }^{22}$ and DECIM-128 [1, 2]. So far, the best known attack on the SG with Krawczyk's parameters is a nearpractical attack in 27]; the best known attack on LILI- $\amalg$ is a distinguishing attack which requires $2^{103}$ bits keystream and $2^{103}$ operations [11]. Previous work 
3, 22, 25] on DECIM focused on the initialization phase which does not contain the ABSG algorithm and there are no known cryptanalytic result on DECIM exploiting the properties of the ABSG decimation. In order to get better attacks on these stream ciphers, we first present an improved correlation analysis of various irregular decimation mechanisms in a unified way, which allows us to get much larger correlation probabilities in these mechanisms than previously known results [16, 26]. Then new correlation attacks are launched against the SG with Krawczyk's parameters, LILI- $\amalg$, DECIM $^{v 2}$ and DECIM-128 to precisely access the security margin of these ciphers. We show that the SG with Krawczyk's parameters is practically insecure. We also show that the initial internal state of LILI- $\amalg$ can be recovered in $2^{72.5}$ operations with a success rate of $93.4 \%$, if $2^{24.1}$-bit keystream and $2^{74.1}$-bit memory are available. This disproves the designers' conjecture that the complexity of any divide-and-conquer attack on LILI- $\amalg$ is in excess of $2^{128}$ operations and requires a large amount of keystream. We also examine the main design idea behind DECIM, i.e., to filter and then decimate the output using the ABSG algorithm, by showing a class of rather large correlations in the ABSG mechanism and mounting attacks faster than exhaustive search on a 160-bit (out of 192-bit) reduced version of DECIM ${ }^{v 2}$ and on a 256-bit (out of 288-bit) reduced version of DECIM-128. We then extend the attack to the full length versions and show that our attack, though slower than exhaustive key search, is $2^{35}$ times faster than the generic time/memory/data tradeoffs. This is the first nontrivial cryptanalytic result on DECIM besides the tricky time/memory/data tradeoffs. While our result confirms the underlying design idea, it shows an interesting fact that the security of DECIM rely more on the length of the involved LFSR than on the ABSG algorithm.

This paper is organized as follows. We first introduce the four target stream ciphers in Section 2. Then an improved correlation analysis of various irregular decimations in a unified way is presented in Section 3. The application of our method to the SG with Krawczyk's parameters, LILI-I, DECIM ${ }^{22}$ and DECIM128 are given in Section 4 respectively. Finally, some conclusions are provided in Section 5 .

\section{Four Irregularly Decimated Stream Ciphers}

The shrinking generator (SG) was proposed in [8] at Crypto'93, which is considered as one of the simplest and strongest stream ciphers currently available. It consists of two LFSR's, say the data LFSR B and the control LFSR S. LFSR $\mathrm{B}$ is irregularly decimated by the regularly clocked LFSR S according to the following rule: the output bit of the data LFSR B is taken iff the current output bit of the control LFSR S is 1. In [19], Krawczyk suggested to use a SG with the following parameters, i.e., LFSR B of length 61 and LFSR S of similar length.

The diagrams of LILI and DECIM are presented in Figure 1. In LILI [ 6, 7], there are two components, i.e., the data generation subsystem and the clock control subsystem. The function $f_{c}$ takes two stages from the regularly clocked LFSR $c$ as inputs and produces an integer $c_{j} \in\{1,2,3,4\}$ which defines the 


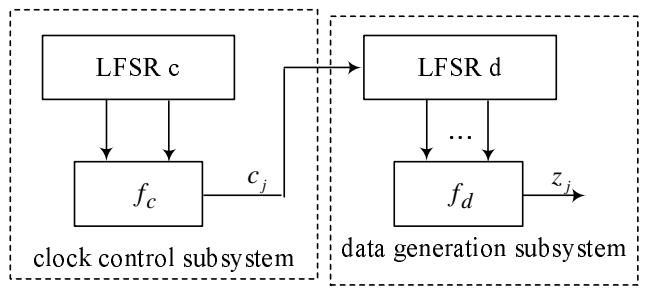

(a) LILI

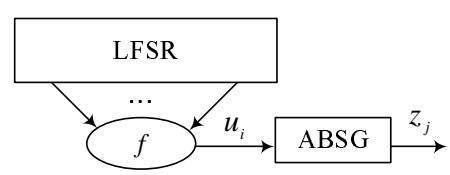

(b) DECIM

Fig. 1. LILI and DECIM

clocking number of LFSR d at time $j$. After the clocking of LFSR d, some stages are taken as inputs to a non-linear boolean function $f_{d}$ to generate the keystream bit. There are two algorithms in this family, i.e., LILI-128 and LILI-I. LILI-Ш is shown to be much stronger than its predecessor LILI-128 in security. We stress here that our target cipher is not the much weaker version LILI-128, but LILI-I.

DECIM contains a unique component in eSTREAM project [1] and is recognized as interesting additions to the field of stream ciphers.

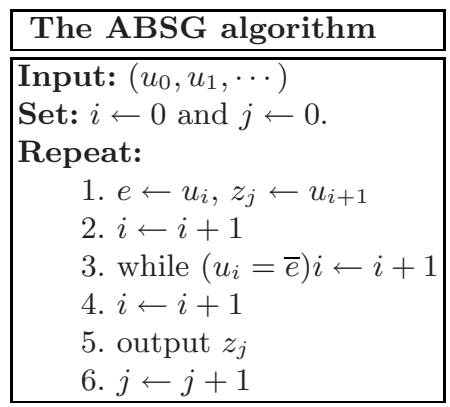

In DECIM, the ABSG algorithm (shown in the block diagram) is used to generate keystream $\left\{z_{j}\right\}$ by irregularly decimating an input stream $\left\{u_{i}\right\}$ from a filter generator. Previous work [3, 22, 25] on DECIM focused on the initialization phase which does not contain the irregular decimation.

DECIM has entered into the third and last phase of the eSTREAM project. The main reason that DECIM is not selected in the final portfolio is its performance compared to other phase 3 hardware candidates. There are no known cryptanalytic result on DECIM exploiting the properties of the ABSG algorithm. Since our results on the latter 3 ciphers are structural cryptanalysis, we only need the relevant parameters of the specific designs, see Table 1.

\section{Correlation Analysis of Arbitrary Irregular Decimation Mechanism}

In this section, we present some theoretical results on arbitrary irregular decimation mechanisms. 
Table 1. Parameters of LILI-II, DECIM ${ }^{v 2}$ and DECIM-128 that are relevant for our structural cryptanalysis

\begin{tabular}{|c||c|c|c|c|}
\hline cipher & $\begin{array}{c}\text { key } \\
\text { size }\end{array}$ & $\begin{array}{c}\text { clock control } \\
\text { LFSR }\end{array}$ & $\begin{array}{c}\text { data generation } \\
\text { LFSR }\end{array}$ & $\begin{array}{c}\text { best linear } \\
\text { approximation }\end{array}$ \\
\hline \hline LILI-I & 128 -bit & 128 -bit & 127 -bit & 0.513671875 \\
\hline DECIM $^{v 2}$ & 80 -bit & - & 192 -bit & 0.5078125 \\
\hline DECIM-128 & 128 -bit & - & 288 -bit & 0.5078125 \\
\hline
\end{tabular}

In irregular decimation, there is an input bit stream $U=\left\{u_{i}\right\}_{i \geq 0}$ and a selection function or rule $\mathcal{D}: i \rightarrow d_{i}$, unknown to the attacker, which defines a nonnegative integer sequence. By applying $\mathcal{D}$ to $\left\{u_{i}\right\}$, another stream $Z=$ $\left\{z_{i}\right\}_{i \geq 0}$ with $z_{i}=u_{\sum_{t=0}^{i} d_{t}}$ is obtained. In practice, the nonnegative integer sequence $\left\{d_{i}\right\}_{i \geq 0}$ may be dependent on $\left\{u_{i}\right\}$, as in the ABSG case.

The starting point of our analysis is the following observation, i.e., any irregular decimation mechanism can be converted into a shrinking-like mechanism. More precisely, given an irregular decimation mechanism, we can construct a bit stream $\left\{s_{i}\right\}_{i \geq 0}$ as follows: for each $i$, let $s_{\sum_{t=0}^{i} d_{t}}=1$, otherwise let $s_{j}=0$. If we regard $\left\{u_{i}\right\}_{i \geq 0}$ as the data source bits, then we have a unified shrinkinglike representation of different irregular decimation mechanisms. Please see the following example.

Example 1. Consider $U=\{0,1,0,1,1,1,0,0,0,0,0,1,0,0,1,0,0,0,0,1,1,0,1,0$, $1,1,1,0\}$. If the ABSG algorithm is applied, we have

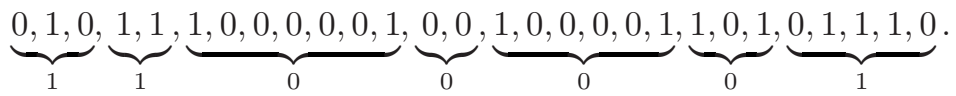

The output is $Z=\{1,1,0,0,0,0,1\}$ and the corresponding $\left\{d_{i}\right\}_{i \geq 0}$ is $\{1,3,2,7,2$, $6,3\}$. If we construct a stream $\left\{s_{i}\right\}_{i \geq 0}$ as $\{0,1,0,0,1,0,1,0,0,0,0,0,0,1,0,1,0,0$, $0,0,0,1,0,0,1,0,0,0\}$, then the shrinking-like representation is

$$
\left\{\begin{array}{l}
s_{i}: 0,1,0,0,1,0,1,0,0,0,0,0,0,1,0,1,0,0,0,0,0,1,0,0,1,0,0,0 \\
u_{i}: 0,1,0,1,1,1,0,0,0,0,0,1,0,0,1,0,0,0,0,1,1,0,1,0,1,1,1,0
\end{array}\right.
$$

It can be easily checked that the output of the shrinking process is $\{1,1,0,0,0$, $0,1\}$.

The advantage of the above viewpoint is that we need not know the actual values of $\left\{s_{i}\right\}$ when determining the data source bits. What we really need is the probabilistic distribution of $\left\{s_{i}\right\}$, which can be determined according to the irregular decimation mechanism by experiments or by theoretical analysis. In the following, we always consider the shrinking-like representation and assume that the distribution of $\left\{s_{i}\right\}$ is known.

Denote the input to the irregular decimation by $\left\{u_{i}\right\}$ and the output keystream by $\left\{z_{j}\right\}$. It follows that $\left\{u_{i}\right\}$ is the data stream in the shrinking-like representation, but we do not know the corresponding $\left\{s_{i}\right\}$. Let $p_{1}=P\left(s_{i}=1\right)$ and 
$p_{0}=P\left(s_{i}=0\right)$, then the index interval in $\left\{z_{j}\right\}$ that $u_{i}$ probably falls into can be determined, i.e., if $u_{i}(i \geq 1)$ (without loss of generality, we assume that $s_{0}=1$ corresponds to $u_{0}$ ) was selected into the keystream, then we have $u_{i}=z_{\sum_{t=0}^{i-1} s_{t}}$. By the central limit theorem, we have $\frac{\sum_{t=0}^{i-1} s_{t}-i \cdot p_{1}}{\sqrt{i \cdot p_{0} p_{1}}} \rightarrow \mathcal{N}(0,1)$, where $\mathcal{N}(0,1)$ is the standard normal distribution. Thus, the probability that the index of $u_{i}$ in the keystream belongs to the interval $I_{\alpha}=\left[i p_{1}-\alpha \cdot \sqrt{i \cdot p_{1} p_{0}}, i p_{1}+\alpha\right.$. $\sqrt{i \cdot p_{1} p_{0}}$ is

$$
P_{I_{\alpha}}=P\left(\sum_{t=0}^{i-1} s_{t} \in I_{\alpha}\right)=\frac{1}{\sqrt{2 \pi}} \int_{-\alpha}^{\alpha} e^{-\frac{x^{2}}{2}} d x .
$$

Let $n_{i}$ be the closest integer to $\frac{2 \alpha \sqrt{i p_{1} p_{0}}-1}{2}$, then there are approximately $2 n_{i}+1$ possible indices in the interval $I_{\alpha}$. Thus, we can count the number of times that 0 and 1 appear in $I_{\alpha}$ and make a majority poll to construct a prediction stream $\left\{\tilde{u}_{i}\right\}$ satisfying $P\left(\tilde{u}_{i}=u_{i}\right)>0.5$. More precisely, let $N_{j}=\#\left\{z_{i} \mid z_{i}=j, i \in I_{\alpha}\right\}$ for $j \in\{0,1\}$ and denote by $\bar{b}$ the complement of a bit $b$, then if $N_{j}>N_{\bar{j}}$, let $\tilde{u}_{i}=j$, otherwise let $\tilde{u}_{i}=\bar{j}$. Thus, we have a predicted stream $\left\{\tilde{u}_{i}\right\}$ of $\left\{u_{i}\right\}$ satisfying the following theorem, which is a generalization of the results in [12, 26].

Theorem 1. For any irregular decimation mechanism, there always exists a correlation between $\left\{\tilde{u}_{i}\right\}$ and $\left\{u_{i}\right\}$, which is given by

$$
P\left(\tilde{u}_{i}=u_{i}\right)=0.5+\frac{p_{1} P_{I_{\alpha}}}{2^{2 n_{i}+1}}\left(\begin{array}{c}
2 n_{i} \\
n_{i}
\end{array}\right) .
$$

Theorem 1, proved in Appendix A, depicts the basic weakness in any irregular decimation mechanism. It is worth noting that in (2), the only a priori knowledge is the distribution of $\left\{s_{i}\right\}$, other elements such as $P_{I_{\alpha}}$ and $n_{i}$ are determined by the choice of $\alpha$. Besides, from (2), we have $0.5<P\left(\tilde{u}_{i}=u_{i}\right) \leq 0.5+\frac{p_{1}}{2}$ for any $i \geq 0$, where the upper bound is achieved when $i=0$. This fact means that the best correlation we can get is determined by the concrete irregular decimation algorithm itself. On the other hand, Theorem 1 shows that $P\left(\tilde{u}_{i}=\right.$ $u_{i}$ ) decreases with $i$ increasing. This is also the bottleneck of previous methods in [12, 16, 26]. To get the best correlation $\max _{\alpha} P\left(\tilde{u}_{i}=u_{i}\right)$, we can pre-compute the optimal values of $\alpha$ for each $i$. Table 2 lists the average correlations, $\sum_{j=0}^{i-1} P\left(\tilde{u}_{j}=\right.$ $\left.u_{j}\right) / i$, we get for some $i$ using the optimal values of $\alpha$ corresponding to the two decimation methods in LILI and DECIM. We made experiments to verify the theoretical values in Table 2 . In LILI- $\amalg$, when $i=10000$, we get the average correlation of 0.518900 for $2^{20}$ randomly chosen initial states of LFSR $\mathrm{c}$ and LFSR d, which is even better than the theoretical estimate.

Table 2. The average biases for some $i$ using the optimum values of $\alpha$

\begin{tabular}{|c||c|c|c|c|c|c|}
\hline$i$ & 1000 & 2000 & 10000 & 20000 & 40000 & 140000 \\
\hline \hline LILI & 0.0318732 & 0.0253801 & 0.0158841 & 0.0131732 & 0.010976 & 0.00794848 \\
\hline ABSG & 0.0273437 & 0.0217052 & 0.0135262 & 0.0112084 & 0.00933383 & 0.00675565 \\
\hline
\end{tabular}


Remarks. Theorem 1 is achieved based on the premise that each bit in $I_{\alpha}$ has the same probability of being correct. In fact, the probability that $u_{i}=z_{r}$ is $P\left(u_{i}=z_{r}\right)=\left(\begin{array}{c}i \\ r\end{array}\right) p_{1}^{r+1} p_{0}^{i-r}$, which are not the same for different $r$. Thus, at least in theory, for a given index interval $I_{\alpha}$, it is better to use the following measure to make a decision: $\Delta_{i}=\sum_{z_{r} \in I_{\alpha}} P\left(z_{r}=u_{i}\right)\left(1-z_{r}\right)-\sum_{z_{r} \in I_{\alpha}} P\left(z_{r}=\right.$ $\left.u_{i}\right) z_{r}=\sum_{z_{r} \in I_{\alpha}}\left(\begin{array}{c}i \\ r\end{array}\right) p_{1}^{r+1} p_{0}^{i-r}\left(1-z_{r}\right)-\sum_{z_{r} \in I_{\alpha}}\left(\begin{array}{c}i \\ r\end{array}\right) p_{1}^{r+1} p_{0}^{i-r} z_{r}$. If $\Delta_{i}>0$, let $\tilde{u}_{i}=0$; otherwise let $\tilde{u}_{i}=1$. However, in this case, we have to compute a large number of binomial coefficients for constructing $\left\{\tilde{u}_{i}\right\}$. To our knowledge, the most efficient method for computing binomial coefficient is by the recursion $\left(\begin{array}{c}i \\ r\end{array}\right)=\left(\begin{array}{c}i \\ r-1\end{array}\right)+\left(\begin{array}{c}i-1 \\ r\end{array}\right)$, which can be computed in about $i^{2}$ time. While in Theorem 1, the time for making a decision is at most linear in $i$. Besides, the probability that we made a correct decision, $P\left(\tilde{u}_{i}=u_{i}\right)$, under the theoretical method is not significantly higher than that in Theorem 1. Our experimental results reveal that the average difference between the two probabilities under the two methods are very small. For example, the gain by the latter method becomes lower than $10^{-4}$ for $i \geq 2^{16}$. Therefore, we adopt the method in Theorem 1.

Next, we take a closer look at the method in Theorem 1. For each $\tilde{u}_{i}$, the validity of the majority poll heavily depends on the distribution of 0 and 1 in the index interval $I_{\alpha}$. Denote by $n_{i, 0}$ and $n_{i, 1}$ the number of 0's and 1 's in $I_{\alpha}$, respectively. Then it is easy to see that the higher the absolute value $\left|n_{i, 0}-n_{i, 1}\right|$, the higher the prediction reliability for $\tilde{u}_{i}$. This motivates us to make a prediction decision only at those positions where $\left|n_{i, 0}-n_{i, 1}\right|$ is larger than a threshold value $\theta$ and otherwise we ignore that position. That is, let

$$
\left\{\begin{array}{l}
\breve{u}_{i}=0, \text { if } n_{i, 0}-n_{i, 1} \geq \theta \\
\breve{u}_{i}=1, \text { if } n_{i, 1}-n_{i, 0} \geq \theta
\end{array}\right.
$$

In this way, we will get a prediction stream $\left\{\breve{u}_{i}\right\}$ at non-consecutive positions, e.g., $\left(\breve{u}_{0}, *, *, \cdots, *, \cdots, \breve{u}_{i}, *, \breve{u}_{i+2}, *, \cdots\right)$. The following theorem shows that the correlation between $\left\{\breve{u}_{i}\right\}$ and $\left\{u_{i}\right\}$ can be guaranteed.

Theorem 2. For any irregular decimation mechanism, if we construct $\left\{\breve{u}_{i}\right\}$ following (3), then the correlation between $\left\{\breve{u}_{i}\right\}$ and $\left\{u_{i}\right\}$ for positions $i$ satisfying $\left|n_{i, 1}-n_{i, 0}\right| \geq \theta$ is lower bounded by

$$
P\left(\breve{u}_{i}=u_{i}\right) \geq 0.5+P_{I_{\alpha}} \cdot \frac{\theta}{2\left(2 n_{i}+1\right)} .
$$

Theorem 2, proved in Appendix B, shows that we can make $P\left(\breve{u}_{i}=u_{i}\right)$ greater than or equal to a value determined by both $\alpha$ and $\theta$. Though we can set a large value of $\theta$ and a large size of $I_{\alpha}$ to get a good enough correlation, the probability that we can find such a segment in the keystream is low. There is a tradeoff between $P\left(\breve{u}_{i}=u_{i}\right)$ and the number of points that having this correlation. This tradeoff is important for the application of Theorem 2.

For simplicity, we denote the prediction stream by $\left\{\tilde{u}_{i}\right\}$ hereafter. In practice, we first set a pointer value $i_{T}$ and for $i>i_{T}$, we use (3) to construct $\left\{\tilde{u}_{i}\right\}$; while 
for $i \leq i_{T}$, we use Theorem 1 to construct $\left\{\tilde{u}_{i}\right\}$. Let $\lceil x\rceil$ be the smallest integer greater than or equal to $x$ and $\lfloor x\rfloor$ be the biggest integer less than or equal to $x$. For $i \geq i_{T}$, let $\mathcal{Z}_{i}=\left(z_{t_{i}}, z_{t_{i}+1}, \cdots, z_{t_{i}+2 n_{i}}\right)$ with $t_{i}=\left\lfloor i p_{1}-\alpha \sqrt{i p_{1} p_{0}}\right\rfloor$. In $(3)$, we actually search for such $\mathcal{Z}_{i}$ that

$$
W_{H}\left(\mathcal{Z}_{i}\right) \geq\left\lceil\frac{2 n_{i}+1+\theta}{2}\right\rceil \text { or } W_{H}\left(\mathcal{Z}_{i}\right) \leq\left\lfloor\frac{2 n_{i}+1-\theta}{2}\right\rfloor
$$

holds, where $W_{H}(\cdot)$ is the hamming weight of the corresponding vector. Let $A_{i}=\left\{\mathcal{Z}_{i} \mid W_{H}\left(\mathcal{Z}_{i}\right) \geq\left\lceil\frac{2 n_{i}+1+\theta}{2}\right\rceil\right.$ or $\left.W_{H}\left(\mathcal{Z}_{i}\right) \leq\left\lfloor\frac{2 n_{i}+1-\theta}{2}\right\rfloor\right\}$. Then the probability that (5) occurs around the position $i$ is the proportion between the cardinality of $\left|A_{i}\right|$ and all the possible values of $\mathcal{Z}_{i}$, which is given approximately by

$$
P_{i}=\frac{2 \cdot \sum_{j=0}^{\left\lfloor\frac{2 n_{i}+1-\theta}{2}\right\rfloor}\left(\begin{array}{c}
2 n_{i}+1 \\
j
\end{array}\right)}{2^{2 n_{i}+1}} \rightarrow 2 \int_{-\frac{\mu_{i}}{\sigma_{i}}}^{\frac{\left\lfloor\frac{2 n_{i}+1-\theta}{2}\right\rfloor-\mu_{i}}{\sigma_{i}}} \frac{1}{\sqrt{2 \pi}} e^{-\frac{x^{2}}{2}} d x
$$

where $\mu_{i}=n_{i}+0.5$ and $\sigma_{i}=\frac{\sqrt{2 n_{i}+1}}{2}$. Since $P_{j}$ is very close to $P_{i}$ if $j$ is close to $i$, for simplicity, we use $P_{i+\left\lfloor\frac{i}{2}\right\rfloor}$ as the average value of $P_{i}$ in the interval $(i, 2 i]$. Accordingly, let $N_{i}$ be the number of points satisfying (3) in $(i, 2 i]$ and we choose the same value of $\alpha$ for the points in $(i, 2 i]$, then we have $i \cdot P_{i+\left\lfloor\frac{i}{2}\right\rfloor} \geq$ $N_{i} \Rightarrow P_{i+\left\lfloor\frac{i}{2}\right\rfloor} \geq \frac{N_{i}}{i}$. This equation indicates that by properly choosing $\theta$, there are at least $N_{i}$ positions constructed in (i,2i] satisfying (3). If $\left\{z_{i}\right\}$ is of length $N_{z}$, then by dividing the whole index interval $\left(i_{T}, N_{z}\right]$ into several segments $\left(i_{T}, 2 i_{T}\right] \cup\left(2 i_{T}, 4 i_{T}\right] \cup \cdots \cup\left(2^{q-1} i_{T}, 2^{q} i_{T}\right]$ with $q=\left\lfloor\log _{2}\left(N_{z}-\alpha \sqrt{N_{z} p_{1} p_{0}}\right)\right\rfloor$, a prediction stream $\left\{\tilde{u}_{i}\right\}_{i \geq i_{T}}$ is constructed segment by segment. In each segment, the average correlation is determined by Theorem 3, proved in Appendix C.

Theorem 3. In $(i, 2 i]$, the average correlation $\left.P\left(\tilde{u}_{i}=u_{i}\right)\right|_{(i, 2 i]}$ can be approximated by $0.5+P_{I_{\alpha}} \cdot \frac{\theta}{4}$, where $\theta=\sqrt{2 n_{i}+1} \cdot \beta$ with $P_{i}=2 \int_{-\infty}^{\beta} \frac{1}{\sqrt{2 \pi}} e^{-\frac{x^{2}}{2}} d x$ specified in (6).

The validity of Theorem 3 is illustrated in Table 3-6. For the SG, LILI-I, DECIM $^{v 2}$ and DECIM-128, we give the theoretical estimates given by Theorem 3 and the corresponding experimental results. For each bias $\varepsilon$ in Table 3-6 and the corresponding $N_{i}$, we verified the result by randomly assigning the initial state of the cipher $2^{20}$ times. We see that the simulation results are very close to the theoretical values.

As we know, the correlations given in Table 3-6 are the largest correlations reported for the four stream ciphers for the corresponding keystream length. For

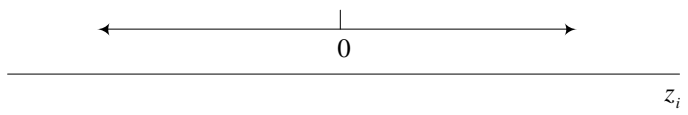

Fig. 2. Constructing $\left\{\tilde{u}_{i}\right\}$ in two directions 
Table 3. The theoretical parameters used for constructing $\left\{\tilde{u}_{i}\right\}$ in SG and the corresponding experimental values

\begin{tabular}{|c||c|c|c|c|c|c|c|}
\hline interval & $\alpha$ & $\theta$ & $P_{i+\left\lfloor\frac{i}{2}\right\rfloor}$ & $\varepsilon$ (Th 3) & $\varepsilon$ (found) & $N_{i}$ (Th 3$)$ & $N_{i}$ (found) \\
\hline$\left(0,2^{11}\right]$ & 1.35 & 11 & $2^{-3}$ & 0.0492344 & 0.059050 & $2^{8}$ & $2^{7.87}$ \\
$\left(2^{11}, 2^{12}\right]$ & 1.35 & 14 & $2^{-3}$ & 0.0384059 & 0.060397 & $2^{8}$ & $2^{7.71}$ \\
$\left(2^{12}, 2^{13}\right]$ & 1.35 & 20 & $2^{-4}$ & 0.0384572 & 0.060687 & $2^{8}$ & $2^{7.60}$ \\
$\left(2^{13}, 2^{14}\right]$ & 1.36 & 27 & $2^{-5}$ & 0.0369315 & 0.055226 & $2^{8}$ & $2^{8.06}$ \\
$\left(2^{14}, 2^{15}\right]$ & 1.345 & 36 & $2^{-6}$ & 0.035035 & 0.054911 & $2^{8}$ & $2^{7.53}$ \\
$\left(2^{15}, 2^{16}\right]$ & 1.36 & 43 & $2^{-6}$ & 0.0293113 & 0.037803 & $2^{9}$ & $2^{8.95}$ \\
$\left(2^{16}, 2^{17}\right]$ & 1.37 & 51 & $2^{-6}$ & 0.0245331 & 0.028811 & $2^{10}$ & $2^{9.95}$ \\
$\left(2^{17}, 2^{18}\right]$ & 1.42 & 61 & $2^{-6}$ & 0.0205909 & 0.024350 & $2^{11}$ & $2^{11.12}$ \\
$\left(2^{18}, 2^{19}\right]$ & 1.43 & 73 & $2^{-6}$ & 0.0172385 & 0.020377 & $2^{12}$ & $2^{12.06}$ \\
$\left(2^{19}, 2^{20}\right]$ & 1.42 & 87 & $2^{-6}$ & 0.014522 & 0.017915 & $2^{13}$ & $2^{12.94}$ \\
$\left(2^{20}, 2^{21}\right]$ & 1.42 & 103 & $2^{-6}$ & 0.0122084 & 0.013745 & $2^{14}$ & $2^{14.03}$ \\
$\left(2^{20}, 2^{21}\right]$ & 1.42 & 122 & $2^{-8}$ & 0.0144604 & 0.015985 & $2^{12}$ & $2^{12.01}$ \\
$\left(2^{21}, 2^{22}\right]$ & 1.39 & 133 & $2^{-7}$ & 0.0112604 & 0.012340 & $2^{14}$ & $2^{13.99}$ \\
\hline
\end{tabular}

Table 4. The theoretical parameters used for constructing $\left\{\tilde{u}_{i}\right\}$ in LILI- $\amalg$ and the corresponding experimental values with $\alpha=1.41$

\begin{tabular}{|c||c|c|c|c|c|c|}
\hline interval & $\theta$ & $P_{i+\left\lfloor\frac{i}{2}\right\rfloor}$ & $\varepsilon$ (Th 3) & $\varepsilon$ (found) & $N_{i}$ (Th 3$)$ & $N_{i}$ (found) \\
\hline$\left(0,2^{11}\right]$ & 11 & $2^{-3}$ & 0.0514225 & 0.0541849 & $2^{8}$ & $2^{7.95}$ \\
$\left(2^{11}, 2^{12}\right]$ & 17 & $2^{-4}$ & 0.0464443 & 0.0550815 & $2^{7}$ & $2^{7.01}$ \\
$\left(2^{12}, 2^{13}\right]$ & 23 & $2^{-5}$ & 0.044389 & 0.0557402 & $2^{7}$ & $2^{7.01}$ \\
$\left(2^{13}, 2^{14}\right]$ & 31 & $2^{-6}$ & 0.042073 & 0.045829 & $2^{7}$ & $2^{6.86}$ \\
$\left(2^{14}, 2^{15}\right]$ & 40 & $2^{-7}$ & 0.038777 & 0.045625 & $2^{7}$ & $2^{7.14}$ \\
$\left(2^{15}, 2^{16}\right]$ & 51 & $2^{-8}$ & 0.0349467 & 0.037282 & $2^{7}$ & $2^{7.49}$ \\
$\left(2^{16}, 2^{17}\right]$ & 65 & $2^{-9}$ & 0.0314334 & 0.042365 & $2^{7}$ & $2^{7.19}$ \\
$\left(2^{17}, 2^{18}\right]$ & 82 & $2^{-10}$ & 0.0281402 & 0.038911 & $2^{7}$ & $2^{7.12}$ \\
\hline
\end{tabular}

Table 5. The theoretical parameters used for constructing $\left\{\tilde{u}_{i}\right\}$ in DECIM D $^{2}$ and the corresponding experimental values with $\alpha=1.41$

\begin{tabular}{|c||c|c|c|c|c|c|}
\hline interval & $\theta$ & $P_{i+\left\lfloor\frac{i}{2}\right\rfloor}$ & $\varepsilon$ (Th 3) & $\varepsilon$ (found) & $N_{i}$ (Th 3) & $N_{i}$ (found) \\
\hline$\left(0,2^{12}\right]$ & 12 & $2^{-3}$ & 0.0413833 & 0.042008 & $2^{9}$ & $2^{8.18}$ \\
$\left(2^{12}, 2^{13}\right]$ & 16 & $2^{-3}$ & 0.0320557 & 0.036443 & $2^{9}$ & $2^{8.89}$ \\
$\left(2^{13}, 2^{14}\right]$ & 19 & $2^{-3}$ & 0.0268251 & 0.026698 & $2^{10}$ & $2^{10.11}$ \\
$\left(2^{14}, 2^{15}\right]$ & 23 & $2^{-3}$ & 0.0231502 & 0.021909 & $2^{11}$ & $2^{11.01}$ \\
$\left(2^{15}, 2^{16}\right]$ & 27 & $2^{-3}$ & 0.0192538 & 0.037282 & $2^{12}$ & $2^{12.05}$ \\
$\left(2^{16}, 2^{17}\right]$ & 32 & $2^{-3}$ & 0.0161431 & 0.015987 & $2^{13}$ & $2^{12.89}$ \\
$\left(2^{17}, 2^{18}\right]$ & 38 & $2^{-3}$ & 0.013526 & 0.013160 & $2^{14}$ & $2^{13.90}$ \\
$\left(2^{18}, 2^{19}\right]$ & 45 & $2^{-3}$ & 0.0113371 & 0.011347 & $2^{15}$ & $2^{15.02}$ \\
\hline
\end{tabular}

the SG, our results are at least 3.6 times larger than those reported in [16]. These large correlations remove the barrier (that the correlation decrease quickly with the keystream length increasing) identified by previous research [16, 26] to a 
Table 6. The theoretical parameters used for constructing $\left\{\tilde{u}_{i}\right\}$ in DECIM-128 and the corresponding experimental values with $\alpha=1.41$

\begin{tabular}{|c||c|c|c|c|c|c|}
\hline interval & $\theta$ & $P_{i+\left\lfloor\frac{i}{2}\right\rfloor}$ & $\varepsilon$ (Th 3) & $\varepsilon$ (found) & $N_{i}$ (Th 3) & $N_{i}$ (found) \\
\hline$\left(2^{11}, 2^{12}\right]$ & 14 & $2^{-3}$ & 0.0392682 & 0.042436 & $2^{8}$ & $2^{7.69}$ \\
$\left(2^{12}, 2^{13}\right]$ & 16 & $2^{-3}$ & 0.0320557 & 0.034141 & $2^{9}$ & $2^{8.89}$ \\
$\left(2^{13}, 2^{14}\right]$ & 27 & $2^{-5}$ & 0.0381199 & 0.048058 & $2^{8}$ & $2^{7.93}$ \\
$\left(2^{14}, 2^{15}\right]$ & 35 & $2^{-6}$ & 0.0352286 & 0.054168 & $2^{8}$ & $2^{8.12}$ \\
$\left(2^{15}, 2^{16}\right]$ & 46 & $2^{-6}$ & 0.0328027 & 0.047282 & $2^{8}$ & $2^{7.76}$ \\
$\left(2^{16}, 2^{17}\right]$ & 55 & $2^{-7}$ & 0.027746 & 0.031999 & $2^{9}$ & $2^{9.29}$ \\
$\left(2^{17}, 2^{18}\right]$ & 65 & $2^{-7}$ & 0.0231366 & 0.026944 & $2^{10}$ & $2^{10.137}$ \\
\hline
\end{tabular}

large extent. Finally, we also need the following theorem to actually construct $\left\{\tilde{u}_{i}\right\}$ in our attack.

Theorem 4. If we choose an intermediate time point to be 0 and construct a prediction stream $\left\{\tilde{u}_{i}\right\}$ in two directions, as shown in the Figure 2. Then $\left\{\tilde{u}_{i}\right\}$ we get will be of double length, while the average correlation will be the same value as if only half of the keystream (one direction) is employed.

Theorem 4 is just an observation which can be verified easily. Note that the results in Table 3-6 are only for one direction.

\section{Applications}

In this section, we use the theoretical results in Section 3 to launch new attacks against the four target ciphers. We need the following notations of the decoding algorithm in [27] involved in our attack.

$-L$ is the length of the involved LFSR.

$-k(k<L)$ is the number of initial state bits to be determined first.

$-t$ is the weight of the parity-checks.

$-n$ is the number of the coefficient patterns appearing in all the parity-checks.

\subsection{The Shrinking Generator}

For the SG with Krawczyk's parameters, we use the correlations specified in Table 3 to mount a correlation attack as follows. Assume we know the feedback polynomial of the data LFSR. We have $\left(2^{7.87}+2^{7.71}+2^{7.60}+2^{8.06}+2^{7.53}+\right.$ $\left.2^{8.95}+2^{9.95}+2^{11.12}+2^{12.06}+2^{12.94}+2^{12.01}\right) \cdot 2=42226$ nonconsecutive bits of $\tilde{u}_{i}$ associated with $u_{i}$ by the relation $P\left(\tilde{u}_{i}=u_{i}\right)=\left(2^{7.87} \cdot 0.559050+2^{7.71} \cdot 0.560397+\right.$ $2^{7.60} \cdot 0.560687+2^{8.06} \cdot 0.555226+2^{7.53} \cdot 0.554911+2^{8.95} \cdot 0.537803+2^{9.95} \cdot 0.528811+$ $\left.2^{11.12} \cdot 0.524350+2^{12.06} \cdot 0.520377+2^{12.94} \cdot 0.517915+2^{12.01} \cdot 0.515985\right) /(42226)=$ 0.521744 . In our case, $L=61$ and we choose the following decoding parameters: $k=25, n=10$ and $t=4$. We construct $10 \cdot \frac{\left({ }^{4226}\right)}{2^{61-25}} \approx 2^{24.21}$ parity-checks with a time complexity of $42226^{2}=2^{30.74}$ operations [20, 27]. Thus, according to [27], 
the time and memory complexities for recovering the internal sate of the data LFSR with a success rate $93 \%$ is $\left(2^{25} \cdot 25+\frac{\left(\begin{array}{l}4226 \\ 2^{61-25}\end{array}\right.}{29} \cdot 29\right) \cdot 10 \approx 2^{33.1}$ operations and $2^{25} \cdot 32+10 \cdot \frac{\left({ }^{42226}\right)}{2^{61-25}} \cdot\left(4 \cdot \log _{2} 42226+61\right) \approx 2^{31.7}$-bit. From Table 3 , the data complexity is $2 \cdot 2^{21}+2 \cdot 1.42 \cdot \sqrt{2^{21} \cdot 0.25}=2^{22.1}$ bits. Note that the attack given in 27] has underestimated the pre-computation complexity, i.e., the true complexity is $2^{39.9}$ operations instead of $2^{33.9}$ operations according to [20]. Thus our attack is the best known attack against the SG with Krawczyk's parameters and shows that it is practically insecure. We have implemented the attack in $\mathrm{C}$ on a computer running under linux. On average, it takes tens of minutes to recover the state of the data LFSR. This is the first reported experimental result on the SG with Krawczyk's parameters.

\subsection{LILI-I}

First note that it is equivalent for the keystream generation if we put the irregular clocking after filtering the regularly clocked LFSR d output. Thus, we can combine $\left\{\tilde{u}_{i}\right\}$ with the linear approximations of $f_{d}$ to get linear approximations on the initial state bits of the LFSR d. First look at the clock control subsystem. We have $c_{j}=f_{c}\left(y_{1}, y_{2}\right)=2 y_{1}+y_{2}+1$, where $y_{1}$ and $y_{2}$ are taken from two stages of LFSR c. If we consider the shrinking-like representation in Section 3 and let $s_{0}=1, s_{\sum_{j=0}^{i-1} c_{j}}=1$ for $i \geq 1$ and other $s_{i}$ 's equal to 0 , then the distribution of $\left\{s_{i}\right\}$ is $P\left(s_{i}=1\right)=\frac{1}{\sum_{j=1}^{4} \frac{1}{4} j}=0.4$ and $P\left(s_{i}=0\right)=0.6$ from the definition of $f_{c}$.

We have computed the Walsh transform of $f_{d}$ in LILI- I, which is listed in Table 7.The Walsh transform values marked with a star are used in our attack, which give $W=236+552+494+364+100+272+78+384=2480$ linear approximations for each bit of $\tilde{u}_{i}$. We first restore the target state of LFSR d, then the corresponding state of LFSR c can be recovered easily, e.g., using the method from [10]. From Table 7, the average correlation between the regularly clocked output of $f_{d}$ and its input is $\left(\frac{3 \cdot 236}{256}+\frac{13 \cdot 100}{1024}+\frac{1 \cdot 552}{128}+\frac{5 \cdot 494}{512}+\frac{11 \cdot 272}{1024}+\frac{7 \cdot 78}{512}+\right.$ $\left.\frac{9 \cdot 364}{1024}+\frac{7 \cdot 384}{1024}\right) / W=0.50926789$. For $\tilde{u}_{i}$, we set $i_{T}=0$ and choose $\alpha=1.41$ which corresponds to $P_{I_{\alpha}}=0.84146$ for all the positions in our estimates. From Table 4 and 8 , the data complexity is $2 \cdot 2^{23}+2 \cdot 1.41 \cdot \sqrt{2^{23} \cdot 0.4 \cdot 0.6}=2^{24.1}$ bits. We divide the index segment $\left(0,2^{23}\right]$ into 13 intervals. The corresponding parameters are listed in Table 4 and 8 . From Table 4 and 8 , there are $2 \cdot 2^{7} \cdot 14=3584$ bits of $\tilde{u}_{i}$ constructed and the average correlation between $\left\{\tilde{u}_{i}\right\}$ and $\left\{u_{i}\right\}$ is $0.5+2^{8} \cdot 0.0514225+2^{7} \cdot(0.0464443+0.042073+0.038777+0.0349467+0.0314334+$

Table 7. The Walsh spectrum of the function $f_{d}$ in LILI- $\amalg$

\begin{tabular}{|c|c|c|c|c|c|}
\hline Value & Number & Value & Number & Value & Number \\
\hline 0 & 280 & $\frac{13}{512}^{*}$ & 100 & $\frac{11}{512}^{*}$ & 272 \\
\hline$\frac{3}{128}$ & 236 & $\frac{5}{512}$ & 256 & $\frac{7}{256}^{*}$ & 78 \\
\hline$\frac{1}{512}$ & 28 & $\frac{3}{512}$ & 132 & $\frac{7}{512}^{*}$ & 384 \\
\hline$\frac{3}{256}$ & 482 & $\frac{1}{64}$ & 552 & $\frac{1}{128}$ & 276 \\
\hline$\frac{1}{256}$ & 162 & $\frac{5}{256}$ & 494 & $\frac{1}{512}$ & 364 \\
\hline
\end{tabular}


Table 8. The parameters for constructing $\left\{\tilde{u}_{i}\right\}$ in LILI- $\amalg$ for longer keystream length

\begin{tabular}{|c|c|c|c|c|}
\hline interval & $\theta$ & $\varepsilon_{2}$ & $P_{i+\left\lfloor\frac{i}{2}\right\rfloor}$ & $N_{i}$ \\
\hline$\left(2^{18}, 2^{19}\right]$ & 103 & 0.0249915 & $2^{-11}$ & $2^{7}$ \\
$\left(2^{19}, 2^{20}\right]$ & 129 & 0.0221166 & $2^{-12}$ & $2^{7}$ \\
$\left(2^{20}, 2^{21}\right]$ & 160 & 0.0194221 & $2^{-13}$ & $2^{7}$ \\
$\left(2^{21}, 2^{22}\right]$ & 199 & 0.0170798 & $2^{-14}$ & $2^{7}$ \\
$\left(2^{22}, 2^{23}\right]$ & 246 & 0.0149264 & $2^{-15}$ & $2^{7}$ \\
\hline
\end{tabular}

$0.0281402+0.0249915+0.0221166+0.0194221+0.0170798+0.0149264) /\left(2^{7}\right.$. $14)=0.5333989$. The folded noise in the final linear approximation is $0.5+$ $2 \cdot 0.0333989 \cdot 0.00926789=0.500619$. The decoding parameters for LILI- $\amalg$ are $t=6, k=60$ and $n=16$. We construct $16 \cdot \frac{\left(\begin{array}{c}2 \cdot 14 \cdot 2^{7} \cdot W \\ 6\end{array}\right)}{2^{127}-60} \approx 2^{66.1}$ parity-checks with a time complexity of $\left(2 \cdot 14 \cdot 2^{7} \cdot W\right)^{3}=2^{69.3}$ operations. Thus, according to [27], the time and memory complexities for recovering the internal sate of LFSR $d$ with a success rate $93.44 \%$ is $\left(2^{60} \cdot 60+\frac{\left(\left(^{2 \cdot 14 \cdot 2^{7} \cdot W}\right)\right.}{6} \cdot 64\right) \cdot 16 \approx 2^{72.32}$ operations and $2^{60} \cdot 32+16 \cdot \frac{\left({ }^{2 \cdot 14 \cdot 2^{7} \cdot W}\right)}{2^{127-60}} \cdot\left(6 \cdot \log _{2}\left(2 \cdot 14 \cdot 2^{7} \cdot W\right)+127\right) \approx 2^{74.1}$-bit. The total time complexity of our attack is $2^{72.32}+2^{69.3} \approx 2^{72.5}$ operations, which is much faster than the exhaustive search for the 128-bit key. Compared to the distinguishing attack in [11], our attack is a state recovery attack with the $2^{79}$ times smaller data complexity and the $2^{31}$ times smaller time complexity. We have implemented the attack on a reduced version of LILI-I, (LFSR $\mathrm{d}$ is of 40-bit), on the same computer as in the SG case. It takes few minutes to recover the state of the 40-bit LFSR d with $2^{16.1}$-bit keystream.

\subsection{DECIM $^{v 2}$ and DECIM-128}

The attack routine is the same as that in LILI- $I$. To construct $\left\{s_{i}\right\}$ for the ABSG mechanism, we let the bit pair corresponding to $(\bar{u}, \bar{u})$ be $(10)$ and let the bit string corresponding to $\left(\bar{u}, u^{i}, \bar{u}\right)$ be $(010 \cdots 0)$ of length $i+2$. Note that we have more choices other than $(010 \cdots 0)$ here, e.g., we can let it to be $(001 \cdots 0)$. It has no effect on the output keystream. For simplicity, we let $\left(\bar{u}, u^{i}, \bar{u}\right)$ be $(010 \cdots 0)$. Since $\frac{1}{\sum_{i=1}^{+\infty} \frac{(1+i)}{2^{i}}}=\frac{1}{3}$, we have $P\left(s_{i}=1\right)=\frac{1}{3}$ and $P\left(s_{i}=0\right)=\frac{2}{3}$ in the ABSG case. In DECIM, the Walsh spectrum of the filter function $f$ has $W=4096$ points having the value $\frac{1}{64}$, others are all 0 .

We also set $i_{T}=0$ and $\alpha=1.41$. First consider the 160 -bit reduced version of DECIM ${ }^{v 2}$ without buffer. From Table 5 and 9 , the data complexity is 2 . $2^{34}+2 \cdot 1.41 \cdot \sqrt{2^{34} \cdot \frac{1}{3} \cdot \frac{2}{3}}=2^{35.1}$ bits. We divide the index segment $\left(0,2^{34}\right]$ into 23 intervals. The corresponding parameters are listed in Table 5 and Table 9. From Table 5 and 9 , there are $2 \cdot\left(2^{9}+\sum_{i=9}^{21} 2^{i}+2^{22} \cdot 8+2^{24}\right)=54525952$ bits of $\tilde{u}_{i}$ constructed and the average correlation between $\left\{\tilde{u}_{i}\right\}$ and $\left\{u_{i}\right\}$, shown in Appendix D, is 0.50267488 . The folded noise is $0.5+2 \cdot 0.0078125 \cdot 0.00267488=$ 0.500042 . The decoding parameters are $t=4, k=66$ and $n=18$. We construct 
Table 9. The parameters for constructing $\left\{\tilde{u}_{i}\right\}$ in $\operatorname{DECIM}^{v 2}$

\begin{tabular}{|l|c|c|c|c||c|c|c|c|c|}
\hline interval & $\theta$ & $\varepsilon_{2}$ & $P_{i+\left\lfloor\frac{i}{2}\right\rfloor}$ & $N_{i}$ & interval & $\theta$ & $\varepsilon_{2}$ & $P_{i+\left\lfloor\frac{i}{2}\right\rfloor}$ & $N_{i}$ \\
\hline$\left(2^{19}, 2^{20}\right]$ & 53 & 0.0094566 & $2^{-3}$ & $2^{16}$ & $\left(2^{27}, 2^{28}\right]$ & 296 & 0.00330107 & $2^{-5}$ & $2^{22}$ \\
$\left(2^{20}, 2^{21}\right]$ & 63 & 0.0079407 & $2^{-3}$ & $2^{17}$ & $\left(2^{28}, 2^{29}\right]$ & 470 & 0.00311483 & $2^{-6}$ & $2^{22}$ \\
$\left(2^{21}, 2^{22}\right]$ & 75 & 0.00668815 & $2^{-3}$ & $2^{18}$ & $\left(2^{29}, 2^{30}\right]$ & 517 & 0.00288294 & $2^{-7}$ & $2^{22}$ \\
$\left(2^{22}, 2^{23}\right]$ & 89 & 0.00561395 & $2^{-3}$ & $2^{19}$ & $\left(2^{30}, 2^{31}\right]$ & 667 & 0.00263001 & $2^{-8}$ & $2^{22}$ \\
$\left(2^{23}, 2^{24}\right]$ & 106 & 0.00472731 & $2^{-3}$ & $2^{20}$ & $\left(2^{31}, 2^{32}\right]$ & 851 & 0.00237274 & $2^{-9}$ & $2^{22}$ \\
$\left(2^{24}, 2^{25}\right]$ & 126 & 0.00397451 & $2^{-3}$ & $2^{21}$ & $\left(2^{32}, 2^{33}\right]$ & 1018 & 0.00212532 & $2^{-10}$ & $2^{22}$ \\
$\left(2^{25}, 2^{26}\right]$ & 149 & 0.00332285 & $2^{-3}$ & $2^{22}$ & $\left(2^{33}, 2^{34}\right]$ & 1034 & 0.00167847 & $2^{-9}$ & $2^{24}$ \\
$\left(2^{26}, 2^{27}\right]$ & 216 & 0.00340647 & $2^{-4}$ & $2^{22}$ & & & & & \\
\hline
\end{tabular}

Table 10. The parameters for constructing $\left\{\tilde{u}_{i}\right\}$ in DECIM-128

\begin{tabular}{|c|c|c|c|c||c|c|c|c|c|}
\hline interval & $\theta$ & $\varepsilon_{2}$ & $P_{i+\left\lfloor\frac{i}{2}\right\rfloor}$ & $N_{i}$ & interval & $\theta$ & $\varepsilon_{2}$ & $P_{i+\left\lfloor\frac{i}{2}\right\rfloor}$ & $N_{i}$ \\
\hline$\left(0,2^{27}\right]$ & 324 & 0.0062582 & $2^{-9}$ & $2^{18}$ & $\left(2^{31}, 2^{32}\right]$ & 665 & 0.00185414 & $2^{-6}$ & $2^{25}$ \\
$\left(2^{27}, 2^{28}\right]$ & 158 & 0.00176206 & $2^{-2}$ & $2^{25}$ & $\left(2^{32}, 2^{33}\right]$ & 869 & 0.00171327 & $2^{-7}$ & $2^{25}$ \\
$\left(2^{28}, 2^{29}\right]$ & 251 & 0.0019793 & $2^{-3}$ & $2^{25}$ & $\left(2^{33}, 2^{34}\right]$ & 1121 & 0.00156276 & $2^{-8}$ & $2^{25}$ \\
$\left(2^{29}, 2^{30}\right]$ & 362 & 0.00201862 & $2^{-4}$ & $2^{25}$ & $\left(2^{34}, 2^{35}\right]$ & 1431 & 0.00141063 & $2^{-9}$ & $2^{25}$ \\
$\left(2^{30}, 2^{31}\right]$ & 498 & 0.00196364 & $2^{-5}$ & $2^{25}$ & & & & & \\
\hline
\end{tabular}

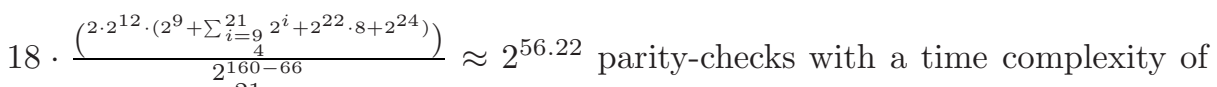
$\left(2 \cdot 2^{12} \cdot\left(2^{9}+\sum_{i=9}^{21} 2^{i}+2^{22} \cdot 8+2^{24}\right)^{2}=2^{77.41}\right.$ operations. Thus, according to [27], the time and memory complexities for recovering the internal sate of the reduced length LFSR with a success rate $92 \%$ is $\left(2^{66} \cdot 66+2^{77.41} \cdot 70\right) \cdot 18 \approx 2^{76.3}$ operations and $2^{66} \cdot 32+18 \cdot 2^{77.41} \cdot\left(8 \cdot \log _{2}\left(2^{9}+\sum_{i=9}^{21} 2^{i}+2^{22} \cdot 8+2^{26}\right)+160\right) \approx 2^{71.3}$-bit.

For the 256-bit reduced version of DECIM-128 without buffer, we set $i_{T}=0$ and $\alpha=1.41$. The data complexity is $2 \cdot 2^{35}+2 \cdot 1.41 \cdot \sqrt{2^{35} \cdot \frac{1}{3} \cdot \frac{2}{3}}=2^{36.1}$ bits. We divide the index segment $\left(0,2^{35}\right]$ into 9 intervals. The corresponding parameters are listed in Table 10 . From Table 10 , there are $2 \cdot\left(2^{25} \cdot 8+2^{18}\right)=$ 537395200 bits of $\tilde{u}_{i}$ constructed and the average correlation between $\left\{\tilde{u}_{i}\right\}$ and $\left\{u_{i}\right\}$ is $0.5+0.0062582 \cdot 2^{18}+0.00176206 \cdot 2^{25}+0.0019793 \cdot 2^{25}+0.00201862$. $2^{25}+0.00196364 \cdot 2^{25}+0.00185414 \cdot 2^{25}+0.00171327 \cdot 2^{25}+0.00156276 \cdot 2^{25}+$ $\left.0.00141063 \cdot 2^{25}\right) /\left(2^{25} \cdot 8+2^{18}\right)=0.50178741$. The folded noise in the final linear approximation is $0.5+2 \cdot 0.0078125 \cdot 0.00178741=0.500028$. The decoding parameters are $t=6, k=112$ and $n=18$. We construct $18 \cdot \frac{\left(\left(^{2 \cdot\left(2^{25} \cdot 8+2^{18}\right)}\right)\right.}{2^{256-112}} \approx 2^{92.53}$ parity-checks with a time complexity of $\left(2 \cdot\left(2^{25} \cdot 8+2^{18}\right)\right)^{3}=2^{123.1}$ operations. Thus, according to [27], the time and memory complexities for recovering the internal sate of the reduced length LFSR in DECIM-128 with a success rate $73.4 \%$ is $\left(2^{112} \cdot 112+2^{92.53} \cdot 118\right) \cdot 18 \approx 2^{122.98}$ operations and $2^{112} \cdot 32+18 \cdot$ $2^{92.53} \cdot\left(6 \cdot \log _{2} 2 \cdot\left(2^{25} \cdot 8+2^{18}\right)+256\right) \approx 2^{117}$-bit. We can extend the above attacks to the full versions of DECIM by guessing the left 32-bit of the state. The results and comparisons with the time/memory/tradeoffs are given in Appendix E. Our results on DECIM confirms the underlying design idea, but shows an interesting 
fact that the security of DECIM rely more on the length of the involved LFSR than on the ABSG algorithm. We implemented the attack on a reduced version of DECIM with 40-bit LFSR. It takes several minutes to restore the state of the LFSR with $2^{18.1}$-bit keystream.

\section{Conclusions}

We presented an improved correlation analysis of arbitrary irregular decimation mechanism and demonstrated the best known attacks on four well-known stream ciphers using irregular decimation. We believe that our correlation analysis can be used to mount efficient attacks against other stream ciphers using irregular decimation, e.g. the alternating step generator and the self-shrinking generator.

Acknowledgements. The author was with State Key Laboratory of Information Security, Institute of Software, Chinese Academy of Sciences, Beijing, 100190, China. This paper is supported by the key programm of the National Natural Science Foundation of China (Grant No. 60833008) and the general programm of the National Natural Science Foundation of China (Grant No. 60603018).

\section{References}

1. Babbage, S., De Cannière, C., Canteaut A., et al.: The eSTREAM portfolio, http://www.ecrypt.eu.org/stream/portfolio.pdf

2. Berbain, C., Billet, O., Canteaut, A., Courtois, N., et al.: DECIM ${ }^{2}$. In: Robshaw, M.J.B., Billet, O. (eds.) New Stream Cipher Designs. LNCS, vol. 4986, pp. 140-151. Springer, Heidelberg (2008)

3. Berbain, C., Gouget, A., Sibert, H.: Understanding Phase Shifting Equivalent Keys and Exhaustive Search, http://eprint.iacr.org/2008/169.ps.gz

4. Canteaut, A., Trabbia, M.: Improved Fast Correlation Attacks Using Parity-Check Equations of Weight 4 and 5. In: Preneel, B. (ed.) EUROCRYPT 2000. LNCS, vol. 1807, pp. 573-588. Springer, Heidelberg (2000)

5. Chose, P., Joux, A., Mitton, M.: Fast Correlation Attacks: An Algorithmic Point of View. In: Knudsen, L.R. (ed.) EUROCRYPT 2002. LNCS, vol. 2332, pp. 209-221. Springer, Heidelberg (2002)

6. Dawson, E., Clark, A., Golić, J., Fuller, J., et al.: The LILI-128 Keystream Generator. In: Stinson, D.R., Tavares, S. (eds.) SAC 2000. LNCS, vol. 2012, pp. 248-261. Springer, Heidelberg (2001)

7. Clark, A., Dawson, E., Fuller, J., Golić, J., et al.: The LILI-Ш Keystream Generator. In: Batten, L.M., Seberry, J. (eds.) ACISP 2002. LNCS, vol. 2384, pp. 25-39. Springer, Heidelberg (2002)

8. Coppersmith, D., Krawczyk, H., Mansour, Y.: The Shrinking Generator. In: Stinson, D.R. (ed.) CRYPTO 1993. LNCS, vol. 773, pp. 22-39. Springer, Heidelberg (1994)

9. Courtois, N.T., Meier, W.: Algebraic Attacks on Stream Ciphers with Linear Feedback. In: Biham, E. (ed.) EUROCRYPT 2003. LNCS, vol. 2656, pp. 345-359. Springer, Heidelberg (2003) 
10. Courtois, N.T.: Fast Algebraic Attacks on Stream Ciphers with Linear Feedback. In: Boneh, D. (ed.) CRYPTO 2003. LNCS, vol. 2729, pp. 176-194. Springer, Heidelberg (2003)

11. Englund, H., Johansson, T.: A New Distinguisher for Clock Controlled Stream Ciphers. In: Gilbert, H., Handschuh, H. (eds.) FSE 2005. LNCS, vol. 3557, pp. 181-195. Springer, Heidelberg (2005)

12. Ekdahl, P., Johansson, T.: Predicting the Shrinking Generator with Fixed Connections. In: Biham, E. (ed.) EUROCRYPT 2003. LNCS, vol. 2656, pp. 330-344. Springer, Heidelberg (2003)

16. Golić, J.D., Mihaljević, M.j.: A Generalized Correlation Attack on a Class of Stream Ciphers Based on the Levenshtein Distance. Journal of Cryptology 3(3), 201-212 (1991)

14. Golić, J.D.: Embedding and Probabilistic Correlation Attacks on ClockedControlled Shift Registers. In: De Santis, A. (ed.) EUROCRYPT 1994. LNCS, vol. 950, pp. 230-243. Springer, Heidelberg (1995)

15. Golić, J.D.: Towards Fast Correlation Attacks on Irregularly Clocked Shift Registers. In: Guillou, L.C., Quisquater, J.-J. (eds.) EUROCRYPT 1995. LNCS, vol. 921, pp. 248-262. Springer, Heidelberg (1995)

16. Golić, J.D.: Correlation Analysis of the Shrinking Generator. In: Kilian, J. (ed.) CRYPTO 2001. LNCS, vol. 2139, pp. 440-457. Springer, Heidelberg (2001)

17. Gouget, A., Sibert, H., Berbain, C., Courtois, N.T., Debraize, B., Mitchell, C.: Analysis of the Bit-Search Generator and Sequence Compression Techniques. In: Gilbert, H., Handschuh, H. (eds.) FSE 2005. LNCS, vol. 3557, pp. 196-214. Springer, Heidelberg (2005)

18. Gouget, A., Sibert, H.: How to Strengthen Pseudo-Random Generators by Using Compression. In: Vaudenay, S. (ed.) EUROCRYPT 2006. LNCS, vol. 4004, pp. 129-146. Springer, Heidelberg (2006)

19. Krawczyk, H.: The Shrinking Generator: Some Practical Considerations. In: Preneel, B. (ed.) FSE 1994. LNCS, vol. 809, pp. 45-46. Springer, Heidelberg (1994)

20. Johansson, T., Jönsson, F.: Fast Correlation Attacks through Reconstruction of Linear Polynomials. In: Bellare, M. (ed.) CRYPTO 2000. LNCS, vol. 1880, pp. 300-315. Springer, Heidelberg (2000)

21. Krause, M.: BDD-Based Cryptanalysis of Keystream Generators. In: Knudsen, L.R. (ed.) EUROCRYPT 2002. LNCS, vol. 2332, pp. 222-237. Springer, Heidelberg (2002)

22. Pasalic, E.: Key Differentiation Attacks on Stream Ciphers, http://eprint.iacr.org/2008/443.pdf

23. Meier, W., Staffelbach, O.: Fast Correlation Attacks on Certain Stream Ciphers. Journal of Cryptology, 159-176 (1989)

24. Molland, H., Helleseth, T.: An Improved Correlation Attack Against Irregular Clocked and Filtered Keystream Generators. In: Franklin, M. (ed.) CRYPTO 2004. LNCS, vol. 3152, pp. 373-389. Springer, Heidelberg (2004)

25. Nakagami, H., Teramura, R., Ohigashi, T., Kuwakado, H.: A Chosen IV Attack Using Phase Shifting Equivalent Keys Against Decim ${ }^{\text {v2, }}$ http://eprint.iacr.org/2008/128.pdf

26. Zhang, B., Wu, H., Feng, D., Bao, F.: A Fast Correlation Attack on the Shrinking Generator. In: Menezes, A. (ed.) CT-RSA 2005. LNCS, vol. 3376, pp. 72-86. Springer, Heidelberg (2005) 
27. Zhang, B., Feng, D.: An Improved Fast Correlation Attack on Stream Ciphers. In: Avanzi, R., Keliher, L., Sica, F. (eds.) SAC 2008. LNCS, vol. 5381, pp. 214-227. Springer, Heidelberg (2009)

\section{A Proof of Theorem 1}

Proof. Note that an irregular decimation mechanism is characterized by the distribution of $\left\{s_{i}\right\}$ in the shrinking-like representation. From the shrinking-like representation and the majority poll, we have

$$
\begin{aligned}
P\left(\tilde{u}_{i}=u_{i}\right)= & \sum_{j=0}^{1} P\left(s_{i}=j\right) P\left(\tilde{u}_{i}=u_{i} \mid s_{i}=j\right)=0.5 p_{0}+p_{1} P\left(\tilde{u}_{i}=u_{i} \mid s_{i}=1\right) \\
= & 0.5 p_{0}+p_{1}\left\{P\left(\sum_{j=0}^{i-1} s_{j} \notin I_{\alpha} \mid s_{i}=1\right) P\left(\tilde{u}_{i}=u_{i} \mid \sum_{j=0}^{i-1} s_{j} \notin I_{\alpha}, s_{i}=1\right)\right. \\
& \left.+P\left(\sum_{j=0}^{i-1} s_{j} \in I_{\alpha} \mid s_{i}=1\right) P\left(\tilde{u}_{i}=u_{i} \mid \sum_{j=0}^{i-1} s_{j} \in I_{\alpha}, s_{i}=1\right)\right\} \\
= & 0.5 p_{0}+0.5 p_{1}\left(1-P_{I_{\alpha}}\right)+p_{1} P_{I_{\alpha}} P\left(\tilde{u}_{i}=u_{i} \mid \sum_{j=0}^{i-1} s_{j} \in I_{\alpha}, s_{i}=1\right),
\end{aligned}
$$

where

$$
\begin{aligned}
P\left(\tilde{u}_{i}=u_{i} \mid \sum_{j=0}^{i-1} s_{j} \in I_{\alpha}, s_{i}=1\right) & =\sum_{b=0}^{1} P\left(u_{i}=b\right) P\left(\tilde{u}_{i}=u_{i} \mid u_{i}=b, \sum_{j=0}^{i-1} s_{j} \in I_{\alpha}, s_{i}=1\right) \\
& =2 \cdot 0.5 \sum_{j=n_{i}}^{2 n_{i}}\left(\begin{array}{c}
2 n_{i} \\
j
\end{array}\right) \frac{1}{2^{2 n_{i}}}=\frac{1}{2}+\frac{1}{2^{2 n_{i}+1}}\left(\begin{array}{c}
2 n_{i} \\
n_{i}
\end{array}\right) .
\end{aligned}
$$

Substituting (8) into (7), we have (2).

\section{B Proof of Theorem 2}

Proof. First note that from (3) and $n_{i, 0}+n_{i, 1}=2 n_{i}+1$, we have

$$
\max \left(n_{i, 0}, n_{i, 1}\right) \geq \frac{2 n_{i}+1+\theta}{2}
$$


for positions satisfying $\left|n_{i, 1}-n_{i, 0}\right| \geq \theta$. Then according to the majority poll and the shrinking-like representation, we have

$$
\begin{aligned}
& P\left(\breve{u}_{i}=u_{i}\right)=P\left(\breve{u}_{i}=u_{i}, \sum_{j=0}^{i-1} s_{j} \notin I_{\alpha}\right)+P\left(\breve{u}_{i}=u_{i}, \sum_{j=0}^{i-1} s_{j} \in I_{\alpha}\right) \\
& \quad=P\left(\sum_{j=0}^{i-1} s_{j} \notin I_{\alpha}\right) P\left(\breve{u}_{i}=u_{i} \mid \sum_{j=0}^{i-1} s_{j} \notin I_{\alpha}\right)+P\left(\sum_{j=0}^{i-1} s_{j} \in I_{\alpha}\right) P\left(\breve{u}_{i}=u_{i} \mid \sum_{j=0}^{i-1} s_{j} \in I_{\alpha}\right) \\
& \quad=\frac{1}{2} \cdot\left(1-P_{I_{\alpha}}\right)+P_{I_{2}} \cdot P\left(\breve{u}_{i}=u_{i} \mid \sum_{j=0}^{i-1} s_{j} \in I_{\alpha}\right) \\
& \quad=\frac{1}{2} \cdot\left(1-P_{I_{\alpha}}\right)+P_{I_{2}} \cdot \frac{\max \left(n_{i, 0}, n_{i, 1}\right)}{2 n_{i}+1} \\
& \geq \frac{1}{2} \cdot\left(1-P_{I_{\alpha}}\right)+P_{I_{2}} \cdot \frac{\frac{2 n_{i}+1+\theta}{2}}{2 n_{i}+1}=0.5+P_{I_{\alpha}} \cdot \frac{\theta}{2\left(2 n_{i}+1\right)} .
\end{aligned}
$$

\section{Proof of Theorem 3}

Proof. From Theorem 2, in the interval $(i, 2 i]$, we have

$$
\begin{aligned}
\left.P\left(\tilde{u}_{i}=u_{i}\right)\right|_{(i, 2 i]} & \geq \frac{\sum_{j \in(i, 2 i]}\left(0.5+P_{I_{\alpha}} \cdot \frac{\theta}{2\left(2 n_{j}+1\right)}\right)}{N_{i}} \\
& \geq 0.5+\frac{1}{N_{i}} \sum_{j \in(i, 2 i]}\left(P_{I_{\alpha}} \cdot \frac{\theta}{2\left(2 n_{j}+1\right)}\right) \\
& =0.5+P_{I_{\alpha}} \cdot \frac{\theta}{2} \sum_{j \in(i, 2 i]}\left(\frac{1}{N_{i}\left(2 n_{j}+1\right)}\right),
\end{aligned}
$$

where $\sum_{j \in(i, 2 i]}$ only sums over the positions satisfying (3). The precise analysis of $\sum_{j \in(i, 2 i]}\left(\frac{1}{N_{i}\left(2 n_{j}+1\right)}\right)$ is complicated in theory. Instead, we use the numerical experiments to determine the value of this term. We made experiments to determine this value in the context of the shrinking generator, LILI- $\amalg$, DECIM $^{\text {2 }}$ and DECIM-128, it turns out that we can take this value as 0.5 . This is illustrated by our experimental results, performed $2^{20}$ times for each $\varepsilon$ and $N_{i}$, which are given in Table 3-6.

In addition, note that the probability in (6) can be rewritten as

$$
2 \int_{-\frac{\mu_{i}}{\sigma_{i}}}^{\frac{\left\lfloor\frac{2 n_{i}+1-\theta}{2}\right\rfloor-\mu_{i}}{\sigma_{i}}} \frac{1}{\sqrt{2 \pi}} e^{-\frac{x^{2}}{2}} d x \approx 2 \int_{-\infty}^{\frac{\left\lfloor\frac{2 n_{i}+1-\theta}{2}\right\rfloor-\mu_{i}}{\sigma_{i}}} \frac{1}{\sqrt{2 \pi}} e^{-\frac{x^{2}}{2}} d x
$$

for $n_{i} \geq 50$. This fact determines the choice of $\beta$. 


\section{Average Correlation in 160-Bit Reduced Version of DECIM $^{v 2}$}

The average correlation between $\left\{\tilde{u}_{i}\right\}$ and $\left\{u_{i}\right\}$ is $0.5+\left(0.0413833 \cdot 2^{9}+0.0320557\right.$. $2^{9}+0.0268251 \cdot 2^{10}+0.0231502 \cdot 2^{11}+0.0192538 \cdot 2^{12}+0.0161431 \cdot 2^{13}+0.013526$. $2^{14}+0.0113371 \cdot 2^{15}+0.0094566 \cdot 2^{16}+0.0079407 \cdot 2^{17}+0.00668815 \cdot 2^{18}+0.00561395$. $2^{19}+0.00472731 \cdot 2^{20}+0.00397451 \cdot 2^{21}+0.00332285 \cdot 2^{22}+0.00340647 \cdot 2^{22}+$ $0.00330107 \cdot 2^{22}+0.00311483 \cdot 2^{22}+0.00288294 \cdot 2^{22}+0.00263001 \cdot 2^{22}+0.00237274$. $\left.2^{22}+0.00212532 \cdot 2^{22}+0.00167847 \cdot 2^{24}\right) /\left(2^{9}+\sum_{i=9}^{21} 2^{i}+2^{22} \cdot 8+2^{24}\right)=0.50267488$.

\section{E Attack Complexity on Full Length Versions of DECIM and Comparisons with TMD}

Note that the complexities of the TMD attack are only rough estimates that ignore the logarithmic factors, while the complexities of our attack are much more accurate values. Our attack is at least $2^{35}$ times faster than the TMD attack in total complexity, while with much smaller data complexity.

Table 11. Attack complexity on full versions of DECIM and comparisons with time/memory/data tradeoff attack

\begin{tabular}{|c|c|c|c|c|c|}
\hline cipher & attack & pre-computation & time & memory & data \\
\hline \hline \multirow{2}{*}{ DECIM $^{v 2}$} & Ours & $2^{77.41}$ & $2^{108.3}$ & $2^{71.3}$ & $2^{35.1}$ \\
\cline { 2 - 6 } & TMD & $O\left(2^{144}\right)$ & $O\left(2^{96}\right)$ & $O\left(2^{96}\right)$ & $O\left(2^{48}\right)$ \\
\hline \hline \multirow{2}{*}{ DECIM-128 8} & Ours & $2^{123.1}$ & $2^{154.98}$ & $2^{117}$ & $2^{36.1}$ \\
\cline { 2 - 6 } & TMD & $O\left(2^{216}\right)$ & $O\left(2^{144}\right)$ & $O\left(2^{144}\right)$ & $O\left(2^{72}\right)$ \\
\hline
\end{tabular}

